\title{
Bone Marrow Aspiration: A Randomized Controlled Trial Assessing the Quality of Bone Marrow Specimens Using Slow and Rapid Aspiration Techniques and Evaluating Pain Intensity
}

\author{
Mette Grønkjær $^{\mathrm{a}}$ Connie F. Hasselgren ${ }^{\mathrm{b}}$ Anne Sofie L. Østergaard ${ }^{\mathrm{b}}$ \\ Preben Johansen ${ }^{c}$ June Korup ${ }^{b}$ Martin Bøgsted $^{b, e}$ Anders E. Bilgrau ${ }^{b, d}$ \\ Paw Jensen ${ }^{b}$ \\ ${ }^{a}$ Clinical Nursing Research Unit, and Departments of ${ }^{b}$ Hematology and ${ }^{c}$ Pathology, Aalborg University Hospital, \\ and Departments of ${ }^{\mathrm{d}}$ Mathematical Sciences and ${ }^{\mathrm{e} C l i n i c a l}$ Medicine, Aalborg University, Aalborg, Denmark
}

\section{Key Words}

Bone marrow aspiration - Bone marrow quality · Pain intensity

\begin{abstract}
Background/Aims: Bone marrow aspiration (BMA) is an essential procedure in the examination of hematological disorders, but there is limited evidence as to whether the aspiration rate affects specimen quality. We aimed to assess the specimen quality and pain intensity using slow (S-technique) or rapid (R-technique) aspiration. Methods: This was a single-center, prospective, randomized patient- and assessorblinded study of 482 patients scheduled for BMA. Specimen quality was evaluated by grading bone marrow (BM) cellularity and counting the number of marrow particles. Pain was assessed using a visual analog scale (VAS). Results: We found a significant difference between the 2 groups with regard to the quality of specimens. For cellularity, the odds ratio (OR) for having a poor quality aspirate using the S-technique versus the R-technique was 3.05 [confidence interval (Cl) 1.795.31]. For BM particles, the quality of specimens with the Stechnique proved to be poor compared with the R-tech-
\end{abstract}

nique (OR 2.52; $\mathrm{Cl}$ 1.51-4.28). We found a statistically significant difference of 1 VAS point $(p<0.001)$ of the median pain intensity in favor of the S-technique. Conclusion: Even though the pain intensity is significantly higher with the R-technique, the median difference is relatively small. We propose that the R-technique is preferable to the S-technique due to better specimen quality. @ $2015 \mathrm{~S}$. Karger AG, Basel

\section{Introduction}

Bone marrow aspiration/biopsy (BMA) is an essential procedure in the initial examination and during the course of hematological disorders. Obtaining the optimal quality of the specimen for pathologic review is obviously important [1]. Textbooks and BMA guidelines rarely mention the aspiration technique, and when they do, rapid aspiration is mentioned [2-4]. Former studies have examined the impact that biopsy devices, operator profession and experience have on the quality of bone marrow (BM) assessment and pain [1,5-10]. To our knowledge, studies on the aspiration technique and its association with spec-

\section{KARGER}

E-Mail karger@karger.com

www.karger.com/aha
C 2015 S. Karger AG, Basel

0001-5792/15/1352-0081\$39.50/0
Paw Jensen

Department of Hematology

Aalborg University Hospital

Mølleparkvej, DK-9000 Aalborg (Denmark)

E-Mail paje@rn.dk 
imen quality are sparse. A randomized, cross-over trial aimed to detect a technique that reduces pain without loss of specimen quality indicated that BMA with low differential pressure tended to be less painful, while maintaining specimen quality [11]. However, that study comprised only a few patients $(\mathrm{n}=49)$ and the study design details are not clear as it is only published as an abstract. Another study concluded that the first $2.5 \mathrm{ml}$ of BM aspirated from one puncture site generated the best specimen quality, but did not consider the aspiration rate [12]. It is well known that BMA is associated with pain and discomfort [2, 13-18]. One study evaluated pain in cancer patients undergoing different types of invasive examinations and found that the highest pain levels were related to procedures such as BMA [19]. A prospective study on procedure-related pain among adult hematologic patients who underwent $\mathrm{BMA}$ found that $70 \%$ reported pain during BMA; $35 \%$ of these reported severe-to-worst-possible pain [14]. Several independent factors are associated with BMA-induced pain such as young age, insufficient information prior to the procedure, previous BMA experience, anticipatory anxiety, long duration and the difficulty of the procedure [15, 20-21]. A recent literature review on strategies for pain reduction during BMA highlighted that research so far has concentrated on pain-associated factors rather than pain-reducing measures [22]. A study assessed pain and anxiety during BMA among patients versus health care professionals and found that registered nurses and physicians underestimated severe pain as well as anxiety for BMA outcome and needle insertion [13]. For patient comfort purposes, it is stressed that patients must be warned of the possibility of suction pain before it occurs and be reassured that it will be brief [23]. It is not known if the intensity of pain is dependent on the aspiration technique. Hence, this study aimed to assess the quality of BM specimens and the overall pain intensity using slow and rapid aspiration techniques. We hypothesized that the proportion of poor-quality specimen samples with the slow aspiration technique (S-technique) is no worse than that of the rapid (R)-technique (primary outcome). In addition, we hypothesized that there is no difference in pain intensity when using the S- and R-techniques (secondary outcome).

\section{Material and Methods}

Study Design and Patients

In this single-center, prospective, patient- and assessor-blinded study, 482 adult patients at the Department of Hematology, Aalborg University Hospital, Denmark, were randomized for either slow aspiration (S-technique) or rapid aspiration (R-technique). Data were collected from November 2012 to September 2013. Patients eligible for inclusion were $>18$ years old and scheduled for BMA. Patients could only be enrolled once. Excluded from participation were patients with altered consciousness, those not able to speak and understand Danish, and those with an international normalized ratio (INR) $>3.5$.

The sample size was based on power calculations using data from a pilot study of 60 consecutive standard BMAs (rapid aspiration) examined for cellularity and the number of marrow particles (where 'no' or 'few' nucleated cells and strictly $<11$ marrow particles were defined as inferior quality). The proportion of accepted biopsies using standard rapid aspiration was $75 \%$ [confidence interval (CI) 64-86] for cellularity and 78.3\% (CI 67-89) for the number of marrow particles. The power calculations used marrow particles as a measure of quality due to the high success rate. We conducted a noninferiority test for the null hypothesis that slow aspiration provides poorer specimen quality than rapid aspiration. Based on the proportion of accepted biopsies, the noninferiority margin was $10 \%$. With a $5 \%$ significance level, a minimum of 241 patients in each aspiration group was needed to reach a power of $80 \%$ for the null hypothesis of no difference in marrow quality.

\section{Data Collection}

The study was integrated in the department's ordinary BMA practice by inviting patients to participate as part of their scheduled BMA. At the Department of Hematology, Aalborg University Hospital, BMAs are conducted by 5 registered nurses (RNs), who have advanced training and several years of experience in performing BMA. Upon informed consent, patients were randomized for either the S-technique or the R-technique via a numbered envelope.

\section{Intervention}

Aspiration of BM was performed using either the S-technique (a slow, low, differential pressure, uniform pull of a 10-ml syringe and aspirating in the same pace as the appearance of the BM for approx. 5-15 s) or the R-technique (a quick pull creating a high differential pressure in a 10-ml syringe for approx. $1 \mathrm{~s}$ and holding a high differential pressure during the aspiration of the marrow). Local anesthesia was used for every patient. The biopsy site was the posterior superior iliac crest. The aspiration technique was blinded for the patient and the pathologist and only known to the RN conducting the BMA. Aspiration and biopsy were performed for all patients. Apart from the difference in aspiration technique, the BMA was performed according to the standard procedure of the department. This involved 2 aspirations from the same puncture site followed by biopsy from another puncture site. From each aspiration, $1.5-3 \mathrm{ml}$ of BM was collected for cytologic preparation, flow cytometry, cytogenetics (upon indication) and bio-banking.

\section{Outcome}

The primary outcome measure was the BM specimen quality. All samples were examined by a single, experienced pathologist who assessed the quality of the specimen from the first aspiration. BM cellularity was graded as either 'no', 'few', 'moderate' or 'many' BM cells. We defined no or few BM cells as inferior quality. The number of marrow particles was graded as $0-25$ and $>25$. We defined $<11$ marrow particles as inferior quality. The secondary outcome measure was the patient's pain intensity. Immediately after 
BMA, patients were asked to assess the overall pain intensity according to a visual analog scale (VAS) which described the patient's pain intensity from 0 to $10(0=$ no pain at all and $10=$ worst pain imaginable). Preprocedural analgesia or anxiolytics were not routinely used.

\section{Statistical Analysis}

Comparisons between groups of observations were carried out using Fisher's exact test for categorical variables and Wilcoxon's rank-sum test for continuous variables at a $5 \%$ significance level. All effect estimates are provided with $95 \%$ one-sided CIs. The analysis of cellularity and BM particles were performed as noninferiority (one-sided) tests for the null hypothesis that the proportion of inferior quality aspirates of the S-technique is no worse than that of the R-technique.

We collected data on diagnoses and grouped the patients into 6 groups: (1) acute leukemia and myelodysplastic syndrome (AL), (2) multiple myeloma (MM), (3) malignant lymphoma and chronic lymphocytic leukemia (MCLL), (4) myeloproliferative disorders (MPD, mastocytosis excluded), (5) other (mainly monoclonal gammopathy of undetermined significance, but also mastocytosis, hemophagocytosis and BM infiltration from other cancer diseases) and (6) normal BM (N). Subanalyses stratified on disease groups were carried out. Analysis of variance (ANOVA) was used to study the association between BM quality and pain and the effects of the explanatory variables. In particular, multiple logistic regression was used to model the association between cellularity and marrow particles and the covariates aspiration technique, disease group, their interaction and RN. Multiple linear regressions were used to model the association between VAS pain intensity and the covariates with aspiration technique, disease, operator, age and sex.

\section{Ethics}

The study was approved by and conducted in compliance with the North Denmark Region Committee on Health Research Ethics (approval No. N-20120058). It was performed according to the Declaration of Helsinki and written informed consent was obtained from all patients before enrolment.

\section{Results}

\section{Patient Characteristics}

A total of 482 patients participated in the study (table 1). There were no significant age and gender difference between the 2 aspiration groups. Of the study population, 11 patients received anxiolytics as premedication ( 4 in the slow-aspiration group and 7 in the rapid-aspiration group). No patients received analgesia as premedication. Patients were allowed to take their usual medicine. In fibrotic marrow specimens it is difficult to estimate cellularity or count the number of marrow particles, so some of these were subsequently excluded. We included $\mathrm{BM}$ fibrosis $(\mathrm{MF})$ grade $0-1$ and excluded samples with MF grade 2-3 (using the WHO grading criteria for $\mathrm{MF}$ ) [24]. Prior to analysis, 38 patients were excluded due to
Table 1. Overview of patient characteristics

\begin{tabular}{|c|c|c|c|}
\hline \multirow[t]{2}{*}{ Characteristics } & \multicolumn{3}{|l|}{ Aspiration } \\
\hline & $\begin{array}{l}\text { slow } \\
(\mathrm{n}=218)\end{array}$ & $\begin{array}{l}\text { rapid } \\
(n=226)\end{array}$ & total \\
\hline \multicolumn{4}{|l|}{ Gender } \\
\hline Female & $79(17.8)$ & $103(23.2)$ & $182(41)$ \\
\hline Male & $139(31.3)$ & $123(27.7)$ & $262(59)$ \\
\hline Age, years & $64.71 \pm 14.11$ & $64.79 \pm 13.62$ & $64.75 \pm 13.85$ \\
\hline \multicolumn{4}{|l|}{ Age group } \\
\hline $18-40$ years & $13(2.9)$ & $12(2.7)$ & $25(5.6)$ \\
\hline $41-50$ years & $23(5.2)$ & $23(5.2)$ & $46(10.4)$ \\
\hline $51-65$ years & $64(14.4)$ & $73(16.4)$ & $137(30.9)$ \\
\hline $66-80$ years & $95(21.4)$ & $94(21.2)$ & $189(42.6)$ \\
\hline$>80$ years & $23(5.2)$ & $24(5.4)$ & $47(10.6)$ \\
\hline \multicolumn{4}{|l|}{ BM cellularity } \\
\hline 0 & $9(2)$ & $1(0.2)$ & $10(2.3)$ \\
\hline $1+$ & $51(11.5)$ & $24(5.4)$ & $75(16.9)$ \\
\hline $2+$ & $87(19.6)$ & $57(12.8)$ & $144(32.4)$ \\
\hline $3+$ & $71(16)$ & $144(32.4)$ & $215(48.4)$ \\
\hline \multicolumn{4}{|l|}{ BM particles } \\
\hline $0-10$ & $59(13.3)$ & $29(6.5)$ & $88(19.8)$ \\
\hline $11-25+$ & $159(35.8)$ & $197(44.4)$ & $356(80.2)$ \\
\hline \multicolumn{4}{|l|}{ Sample usable } \\
\hline Yes & $214(48.2)$ & $225(50.7)$ & 439 (98.9) \\
\hline No & $4(0.9)$ & $1(0.2)$ & $5(1.1)$ \\
\hline \multicolumn{4}{|l|}{ Dry BM } \\
\hline Yes & 0 & $3(0.7)$ & $3(0.7)$ \\
\hline No & $218(49.1)$ & $223(50.2)$ & $441(99.3)$ \\
\hline \multicolumn{4}{|l|}{ BM infiltration } \\
\hline Yes & $42(9.5)$ & $31(7)$ & $73(16.4)$ \\
\hline No & $176(39.6)$ & $195(43.9)$ & $371(83.6)$ \\
\hline \multicolumn{4}{|l|}{ Diagnosis class } \\
\hline $\mathrm{AL}$ & $17(3.8)$ & $15(3.4)$ & $32(7.2)$ \\
\hline Other & $10(2.3)$ & $19(4.3)$ & $29(6.5)$ \\
\hline MCLL & $32(7.2)$ & $37(8.4)$ & $69(15.6)$ \\
\hline MM & $21(4.7)$ & $10(2.3)$ & $31(7)$ \\
\hline MPD & $20(4.5)$ & $18(4.1)$ & $38(8.6)$ \\
\hline MPD and MCLL & 0 & $1(0.2)$ & $1(0.2)$ \\
\hline $\mathrm{N}$ & $117(26.4)$ & $126(28.4)$ & $243(54.9)$ \\
\hline
\end{tabular}

Values express $n(\%)$ or mean \pm SD.

hypoplasia/aplasia $(n=5)$, fibrosis $(n=13)$, samples lost/ destroyed due to an error during tissue processing at the Department of Pathology $(\mathrm{n}=10)$, hairy-cell leukemia (dry tap; $\mathrm{n}=3)$, BM necrosis $(\mathrm{n}=2)$, osteosclerotic metastatic carcinoma $(\mathrm{n}=2)$, information lost $(\mathrm{n}=2)$ and other biopsy needles used ( $\mathrm{n}=1$; fig. 1 ). 
Fig. 1. Flowchart of the study.

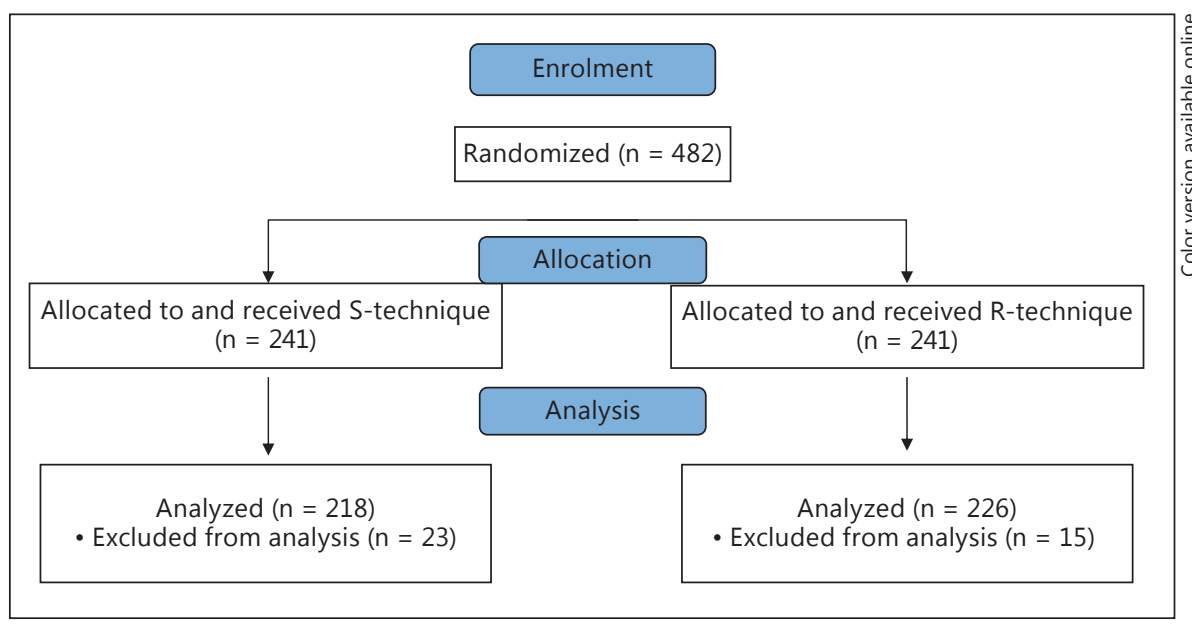

Fig. 2. Observed frequencies of BM particles and cellularity for the S- and R-techniques.

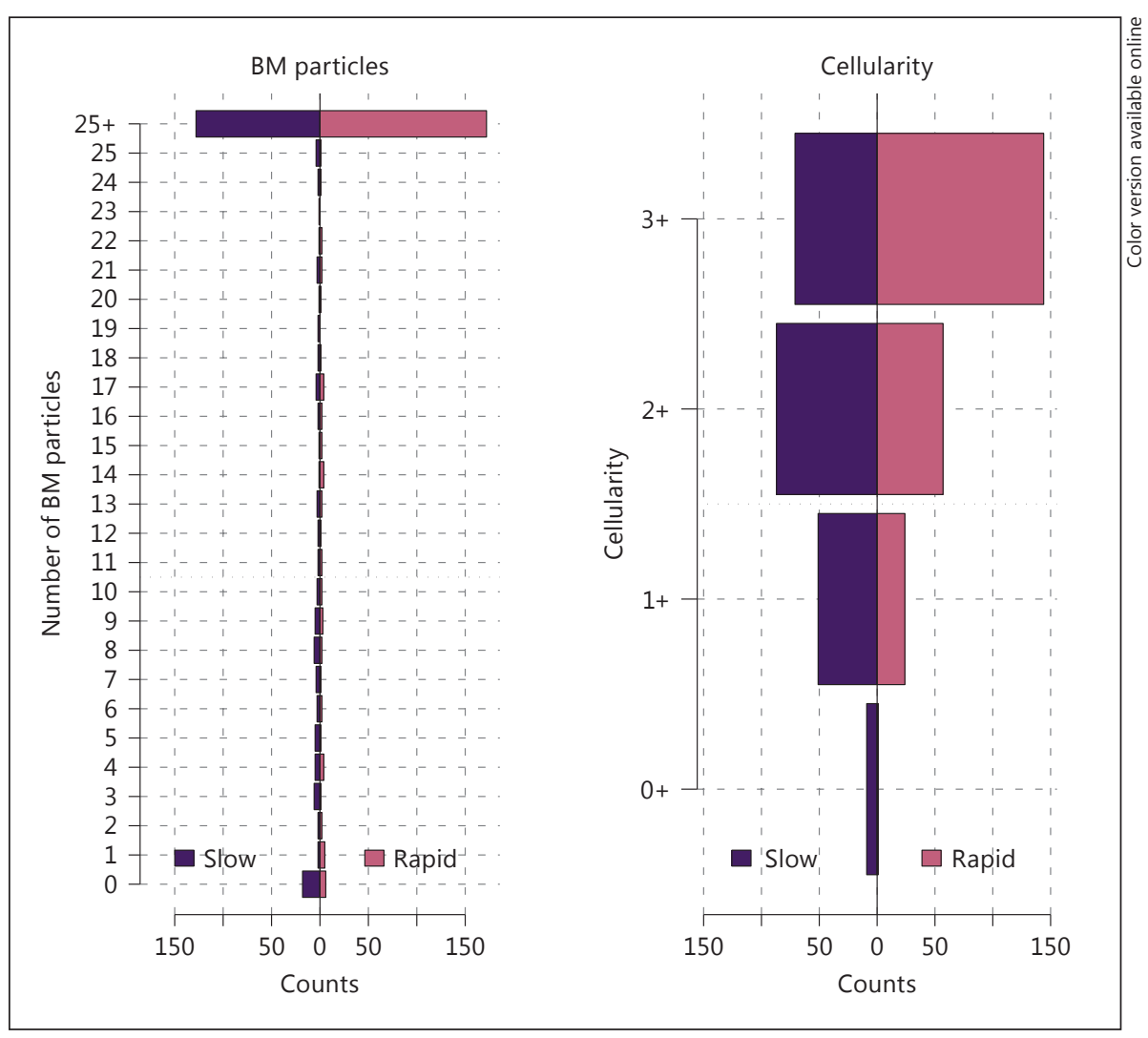

\section{Bone Marrow Quality}

We found a significant difference in the quality of the specimen between aspiration techniques. For BM cellularity, the odds ratio (OR) for having a poor-quality aspirate using the S-technique compared to the R-technique was 3.05 (CI 1.79-5.31, p < 0.001; fig. 2; table 2). For marrow particles, the OR for having a poor-quality aspirate using the S- versus the R-technique was 2.52 (CI 1.51-4.28, p <
0.001; table 2). The pathologist assessed whether the specimen as a whole (including blood, biopsy and aspirated marrow) was usable. For this question, there was no statistically significant difference (OR 4.19, $\mathrm{p}=0.208$ ) between the 2 groups, although there was a trend towards better quality using the R-technique (table 2). According to the disease groups, the significant differences were only retained in the MCLL and N groups (table 2). From the 


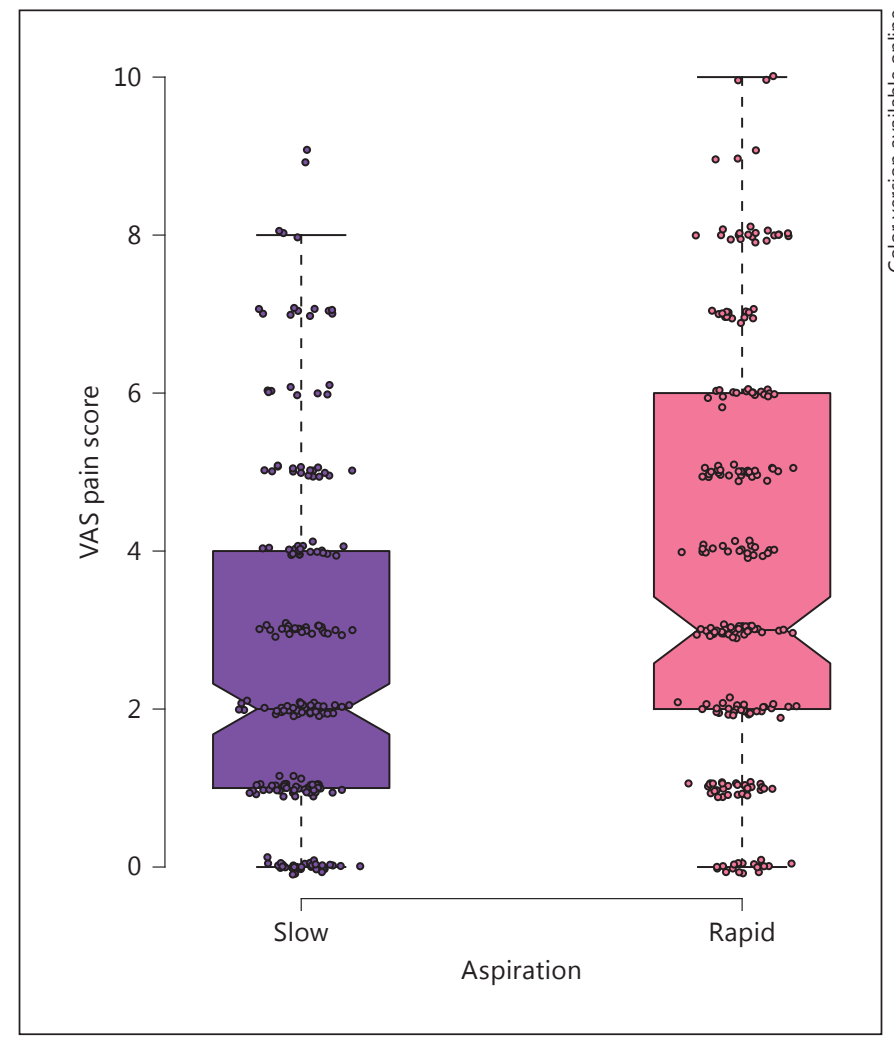

Fig. 3. Box plots of the VAS pain score for the S- and R-techniques.

logistic regression and corresponding ANOVA (online suppl. table S2; for all online suppl. material, see www. karger.com/doi/10.1159/000438480), only the aspiration technique was significant for both measures of sample quality (particles: $\mathrm{p}<0.001$ and cellularity: $\mathrm{p}<0.001$ ). The $\mathrm{RN}$ effect was significant only for the BM particles ( $\mathrm{p}=$ 0.03 ). There was no significant effect of disease group or interaction between disease group and aspiration technique (online suppl. table S1). The significant RN effect prompted further investigation in terms of RN experience. The 5 RNs were categorized as having more or less experience at the time of the aspiration from the date of employment. The level of experience was defined by a threshold of 3 years. The RNs have all approximately been performing 6 aspirations per day once a week since the date of employment. By Fisher's exact test, the experience level of the $\mathrm{RN}$ was independent of the sample quality by both measures (cellularity: $\mathrm{p}=0.901$ and BM particles: $\mathrm{p}=0.806$ ).

\section{Pain Intensity}

The median VAS was 2 and 3, using the S-technique and R-technique, respectively (fig. 3). The difference of 1
Table 2. Fisher's exact test for BM particles and cellularity for all patients, stratified according to disease group

\begin{tabular}{lllllll}
\hline Response & \multicolumn{2}{l}{ Odds } & OR & $95 \%$ CI & $\begin{array}{l}\mathrm{p} \\
\text { value }\end{array}$ \\
\cline { 2 - 3 } & slow & rapid & & & \\
\hline All patients & & & & & \\
Particles & 0.37 & 0.15 & 2.515 & $1.51-4.28$ & $<0.001$ \\
Cellularity & 0.38 & 0.12 & 3.046 & $1.79-5.31$ & $<0.001$ \\
Nonusable sample & 0.02 & 0 & 4.193 & $0.41-207.81$ & 0.208 \\
\hline Disease & group & & & & & \\
AL & Particles & 0.55 & 0.25 & 2.129 & $0.35-16.45$ & 0.444 \\
& Cellularity & 0.7 & 0.36 & 1.886 & $0.35-11.67$ & 0.472 \\
MCLL & Particles & 0.68 & 0.19 & 3.579 & $1.06-13.55$ & 0.030 \\
& Cellularity & 0.39 & 0.09 & 4.467 & $0.98-28.39$ & 0.053 \\
MM & Particles & 0.17 & 0.11 & 1.482 & $0.1-87.33$ & 1 \\
& Cellularity & 0.4 & 0 & Inf & $0.61-$ Inf & 0.141 \\
MPD & Particles & 0.33 & 0.27 & 1.243 & $0.22-7.59$ & 1 \\
& Cellularity & 0.43 & 0.27 & 1.588 & $0.3-9.38$ & 0.716 \\
N & Particles & 0.33 & 0.12 & 2.626 & $1.26-5.72$ & 0.007 \\
& Cellularity & 0.33 & 0.12 & 2.626 & $1.26-5.72$ & 0.007 \\
Other & Particles & 0.25 & 0.12 & 2.066 & $0.13-33.44$ & 0.592 \\
& Cellularity & 0.25 & 0.06 & 4.249 & $0.19-279.29$ & 0.267 \\
\hline
\end{tabular}

Inf $=$ Infinity.

Table 3. Median VAS pain intensity and Wilcoxon's rank sum test for all patients and stratified into disease groups

\begin{tabular}{lllr}
\hline & \multicolumn{2}{l}{ Median VAS } & \multirow{2}{*}{ p value } \\
\cline { 2 - 3 } & slow & rapid & \\
\hline All patients & 2 & 3 & $<0.001$ \\
\hline Disease group & & & \\
AL & 2 & 3.5 & 0.173 \\
Other & 1 & 4 & 0.001 \\
MCLL & 2 & 3 & 0.016 \\
MM & 3 & 3.5 & 0.047 \\
MPD & 2.5 & 4 & 0.038 \\
N & 2 & 3 & $<0.001$ \\
\hline
\end{tabular}

VAS point between the S- and R-techniques in median pain intensity was small, but was statistically significant $(\mathrm{p}<0.001)$ in favor of the S-technique (table 3$)$.

The statistical significance was retained in all but the AL patient subgroup (table 3 ). The ANOVA analysis also yielded strong evidence for an effect of the aspiration technique ( $\mathrm{p}<0.001)$. Here, we found mild evidence $(\mathrm{p}=$ 0.044 ) that VAS pain decreases with age (online suppl. table S2). Furthermore, the data are consistent with no 
effect of disease group, $\mathrm{RN}$ and gender on the VAS pain intensity as well as no interacting effect between disease group and aspiration technique (online suppl. table S2).

\section{Discussion}

From the measurements of the BM cellularity and particles, we found evidence that the R-technique generated a better specimen quality than the S-technique. Pain intensity was significantly higher when using the R-technique compared to the S-technique. The increase of 1 in median VAS pain intensity, however, can be considered small compared to the variability of the VAS in each group (fig. 2). Analysis according to disease group showed the same tendency as for the overall analysis, but was only significant in the MCLL and N groups. The nonsignificance of the remaining groups may be a matter of low power due to small subgroups. Similarly, the ANOVA analysis also suggested that the impact of the aspiration technique on sample quality is unchanged within each disease group. In other words, the effect of the aspiration technique was independent of the disease status. The exclusion of MF grade 2-3 may potentially bias the subgroup of MPD. As the results regarding BM quality and pain from this subgroup were in line with the other subgroups, we believe the potential bias was minimal and had no impact on the overall results.

We found a clear difference in median pain across patients. This observation is in agreement with the Japanese study in which BMA with low-differential pressure tended to be less painful [11]. The same study, however, indicated that slow suctioning did not affect the quality of the specimen, which is not supported by our study. This inconsistence may be due to a fairly small sample size in the Japanese study. Although the pain intensity was higher among patients in the R-technique group, the relatively small difference of 1 on the VAS convinced us to attribute more importance to specimen quality than pain intensity. It is, however, important to acknowledge that all patients are different and that pain is a subjective phenomenon. Moreover, patients with hematological disorders often have repeated BMAs to determine their disease trajectory, treatment efficacy and recovery process. Former studies show a clear association between pain intensity, previous BMAs and inadequate information $[16,20,25]$. In patients who require repeated BMAs, fear and anticipatory pain for this procedure can cause great distress [26]. The multitude of factors besides the aspiration technique involved in the perceived pain intensity highlights the im- portance of taking the individual into consideration before and during BMA.

For this study, we included all eligible adult patients who were scheduled for a BMA. The aim was to conduct a study that was as identical to the daily clinical work as possible. The BMA procedure at Aalborg University Hospital is performed by $5 \mathrm{RNs}$ with advanced training. The feasibility of the study was dependent on the daily practice of the department including staffing and work schedules, and thus all 5 RNs were involved in the data collection. They were trained to follow the same procedure when informing the patients, obtaining consent and performing the randomization dependent on BMA. For practical reasons, it was not possible to ensure blinding of the $\mathrm{RN}$ who asked the standardized questions. Although we have ensured uniform information, BMA procedure and pain assessment, we cannot rule out the risk of potential bias caused by minor differences between the RNs. The blinding was complete for the pathologist regarding the evaluation of the quality parameters. For the pain assessment, the patients were blinded for the aspiration technique when scoring the intensity of pain using the VAS. The use of VAS for assessing pain intensity can be questioned as it is predominantly used for comparing pain assessment in individuals rather than between groups, which creates a potential bias regarding the measurement of pain. However, the absence of appropriate pain assessment scales, the frequent use of VAS in similar studies and the large sample size justify the use of VAS in this setting [27]. The fact that our study included more men than women may be owing to the fact that more men than women suffer from hematological illness [28]. We do not anticipate that gender influences specimen quality. Seeing that there was no difference on pain measurement between men and women, we believe that gender imbalance does not affect the main conclusions. We acknowledge that the single-center study design is a weakness that may influence the external validity of our findings. Widespread changes in clinical practice generally require results from multi-center studies in order to validate the results in different settings.

In conclusion, the quality of BMA using the R-technique for aspirating BM is better than when using the Stechnique. The pain intensity is significantly higher when using the R-technique than with the S-technique. However, the difference in pain intensity is only moderate compared to the general variability of the VAS pain score. Even though there is a difference in pain intensity, we believe that preference should be given to the R-technique in BMA due to the better specimen quality.
86

Acta Haematol 2016;135:81-87 DOI: 10.1159/000438480
Grønkjær/Hasselgren/Østergaard/ Johansen/Korup/Bøgsted/Bilgrau/Jensen 


\section{Acknowledgements}

We would like to thank all the patients for their willingness to participate in the study.

\section{Disclosure Statement}

All authors declare that there were no conflicts of interest.

\section{References}

1 Odejide OO, Cronin AM, DeAngelo DJ, Bernazzoli ZA, Jacobson JO, Rodig SJ, LaCasce AS, Mazeika TJ, Earles KD, Abel GA: Improving the quality of bone marrow assessment: impact of operator techniques and use of a specimen preparation checklist. Cancer 2013; 119:3472-3478.

2 Choby BA: Bone marrow aspiration and biopsy; in Pfenninger JL, Fowler GC (eds): Pfenninger and Fowler's Procedures for Primary Care, ed 3. Elsevier, 2011, pp 14041408 .

3 Zehnder J: Bone marrow aspiration and biopsy: indications and technique. UptoDate 2015. http://www.uptodate.com/contents/ bone-marrow-aspiration-and-biopsy-indications-and-technique? source=search_result \& search=bone+marrow+biopsy\&selectedTitle $=1 \% 7 \mathrm{E} 150$ (accessed March 26, 2015).

4 Malempati S, Joshi S, Lai S, Braner D, Tegtmeyer K: Videos in clinical medicine. Bone marrow aspiration and biopsy. N Engl J Med 2009;361:e28.

5 Swords RT, Anguita J, Higgins RA, Yunes AC, Naski M, Padmanabhan S, Kelly KR, Mahalingam D, Philbeck T, Miller L, Puga TA, Giles FJ, Kinney MC, Brenner AJ: A prospective randomised study of a rotary powered device (OnControl) for bone marrow aspiration and biopsy. J Clin Pathol 2011;64:809-813.

6 Swords RT, Kelly KR, Cohen SC, Miller LJ, Philbeck TE, Hacker SO, Spadaccini CJ, Giles FJ, Brenner AJ: Rotary powered device for bone marrow aspiration and biopsy yields excellent specimens quickly and efficiently. J Clin Pathol 2010;63:562-565.

7 Bucher CM, Lehmann T, Tichelli A, Tzankov A, Dirnhofer S, Passweg J, Rovó A: Comparison of a powered bone marrow biopsy device with a manual system: results of a prospective randomised controlled trial. J Clin Pathol 2013;66:24-28.

8 Bain BJ: Bone marrow trephine biopsy. J Clin Pathol 2001;54:737-742.
9 Lee SH, Erber WN, Porwit A, Tomonaga M, Peterson LC: ICSH guidelines for the standardization of bone marrow specimens and reports. Int J Lab Hematol 2008;30:349-364.

10 Berenson JR, Yellin O, Blumenstein B, Bojanower D, Croopnick J, Aboulafia D, Upadhyaya G, Spadaccini C: Using a powered bone marrow biopsy system results in shorter procedures, causes less residual pain to adult patients, and yields larger specimens. Diagn Pathol 2011:6:23.

11 Asano-Mori Y, Mori S, Ueti T, Sakai M, Yamashita T, Ohashi K, Akiyama H, Sakamaki $\mathrm{H}$ : Painless bone marrow aspiration by slow suctioning do not effect peripheral blood contamination to the specimen: randomized prospective crossover trials. Haematologica 2009;94:453-454.

12 Helgestad J, Rosthoj S, Johansen P, Varming $\mathrm{K}$, Ostergaard E: Bone marrow aspiration technique may have an impact on therapy stratification in children with acute lymphoblastic leukaemia. Pediatr Blood Cancer 2011; 57:224-226.

13 Liden Y, Olofsson N, Landgren O, Johansson E: Pain and anxiety during bone marrow aspiration/biopsy: comparison of ratings among patients versus health-care professionals. Eur J Oncol Nurs 2012;16:323-329.

14 Liden Y, Landgren O, Arner S, Sjolund KF, Johansson E: Procedure-related pain among adult patients with hematologic malignancies. Acta Anaesthesiol Scand 2009;53:354363.

15 Vanhelleputte P, Nijs K, Delforge M, Evers G, Vanderschueren S: Pain during bone marrow aspiration: prevalence and prevention. J Pain Symptom Manage 2003;26:860-866.

16 McGrath P, Rawson-Huff N, Holewa H: Procedural care for adult bone marrow aspiration and biopsy: qualitative research findings from Australia. Cancer Nurs 2013;36:309-316.

17 Trewhitt KG: Bone marrow aspiration and biopsy: collection and interpretation. Oncol Nurs Forum 2001;28:1409-1415.
18 Kuball J, Schuts J, Gamm H, Weber M: Bone marrow punctures and pain. Acute Pain 2004 6:9-14.

19 Portnow J, Lim C, Grossman SA: Assessment of pain caused by invasive procedures in cancer patients. J Natl Compr Canc Netw 2003; 1 435-439.

20 Degen C, Christen S, Rovo A, Gratwohl A: Bone marrow examination: a prospective survey on factors associated with pain. Ann $\mathrm{He}$ matol 2010;89:619-624.

21 Riley RS, Hogan TF, Pavot DR, Forysthe R, Massey D, Smith E, Wright L Jr, Ben-Ezra J: A pathologist's perspective on bone marrow aspiration and biopsy. I. Performing a bone marrow examination. J Clin Lab Anal 2004; 18:70-90.

22 Hjortholm N, Jaddini E, Halaburda K, Snarski E: Strategies of pain reduction during the bone marrow biopsy. Ann Hematol 2013;92: 145-149.

23 Bain BJ: Bone marrow aspiration. J Clin Pathol 2001;54:657-663.

24 Swerdlow SH, Campo E, Harris NL, Jaffe ES, Pileri SA, Stein H, Thiele J, Vardiman JW: WHO Classification of Tumours of Haematopoietic and Lymphoid Tissues, ed 4. Lyon, IARC, 2008.

25 Watmough S, Flynn M: A review of pain management interventions in bone marrow biopsy. J Clin Nurs 2011;20:615-623.

26 Johnson H, Burke D, Plews C, Newell R, Parapia L: Improving the patient's experience of a bone marrow biopsy - an RCT. J Clin Nurs 2008; 17:717-725.

27 Bijur P, Silver W, Gallagher J: Reliability of the visual analog scale for measurement of acute pain. Acad Emerg Med 2001;8:1153-1157.

28 Müller A, Ihorst G, Mertelsmann R, Engelhardt M: Epidemiology of non-Hodgkin's lymphoma (NHL): trends, geographic distribution, and etiology. Ann Hematol 2005;84 $1-12$. 